\title{
Pitch-angle scattering in pure two-dimensional and two-component turbulence
}

\author{
A. Shalchi ${ }^{1}$, J. W. Bieber ${ }^{2}$, and W. H. Matthaeus ${ }^{2}$ \\ ${ }^{1}$ Institut für Theoretische Physik, Lehrstuhl IV: Weltraum- und Astrophysik, Ruhr-Universität Bochum, 44780 Bochum, Germany \\ e-mail: andreasm4@yahoo.com \\ 2 Bartol Research Institute and Department of Physics and Astronomy, University of Delaware, Newark, DE 19716, USA
}

Received 19 November 2007 / Accepted 25 February 2008

\section{ABSTRACT}

\begin{abstract}
Aims. It has been demonstrated that quasilinear theory is inappropriate for describing pitch-angle scattering and parallel spatial diffusion, if a turbulence model with a strong two-dimensional component is assumed. We aim to revisit this problem using a more systematic and reliable approach.

Methods. We consider particle transport in pure two-dimensional turbulence, by using a vector-potential description of pitch-angle scattering.

Results. It is demonstrated that pitch-angle scattering in pure two-dimensional turbulence behaves subdiffusively and, thus, parallel scattering is expected to behave superdiffusively. In the slab/2D composite model, the total pitch-angle Fokker-Planck coefficient reaches the slab limit when very long timescales are considered.
\end{abstract}

Key words. diffusion - turbulence - scattering

\section{Introduction}

The standard approach for understanding and describing charged particle scattering by magnetic turbulence is the application of quasilinear theory (QLT, Jokipii 1966). However, a variety of nonlinear theories for cosmic-ray diffusion have been developed in the years after QLT was proposed (e.g. Völk 1973; Owens 1974; Jones et al. 1973, 1978; Goldstein 1976), and more recently (e.g. Matthaeus et al. 2003; Shalchi et al. 2004b; Shalchi 2005, 2006, Shalchi \& Kourakis 2007; Le Roux \& Webb 2007). Although some of these theories are extensions to QLT, some others provide completely independent approaches to the theoretical description of particle-scattering.

Although it is known and accepted that QLT fails to describe particle scattering perpendicular to the mean magnetic field $\boldsymbol{B}_{0}=$ $B_{0} \boldsymbol{e}_{z}$, several authors assume that QLT can be used to describe parallel scattering. For example, Bieber et al. (1994) and more recently Shalchi et al. (2006) have shown that a combination of QLT and an improved turbulence model, which includes the steepening of the turbulence spectrum at high wavenumber, dynamical turbulence effects, and spectral anisotropy, can reproduce parallel mean free paths of cosmic-rays inferred from solar energetic particle studies (see also Dröge 2003; Shalchi \& Schlickeiser 2004).

In such improved turbulence models it has been assumed that real turbulence can be approximated by a superposition of so-called slab modes, for which the turbulent fields vary only along the mean field $\delta \boldsymbol{B}(\boldsymbol{x})=\delta \boldsymbol{B}(z)$, and two-dimensional modes, for which the turbulent fields vary only in the two directions perpendicular to the mean field $\delta \boldsymbol{B}(\boldsymbol{x})=\delta \boldsymbol{B}(x, y)$. A key effect of the reproduction of observed parallel scattering mean free paths is vanishing pitch-angle scattering in pure two-dimensional turbulence. In the two-component model, or slab/2D composite model, the pitch-angle scattering coefficient, or pitch-angle Fokker-Planck coefficient, can also be written as a superposition of a slab and a two-dimensional coefficient:

$D_{\mu \mu}^{\mathrm{comp}}=D_{\mu \mu}^{\mathrm{slab}}+D_{\mu \mu}^{2 \mathrm{D}}$.

By applying the QLT result $D_{\mu \mu}^{2 \mathrm{D}}=0$ and

$D_{\mu \mu}^{\text {slab }} \sim\left(\frac{\delta B_{\text {slab }}}{B_{0}}\right)^{2}$

pitch-angle scattering is directly proportional to the energy of the slab modes $\sim \delta B_{\text {slab }}^{2} / B_{0}^{2}$.

By converting from pure-slab turbulence $\left(\delta B_{\text {slab }}^{2} / \delta B^{2}=1\right)$, to a two-component model, for which $20 \%$ of the energy is in slab modes and $80 \%$ in two-dimensional modes $\left(\delta B_{\text {slab }}^{2} / \delta B^{2}=0.2\right)$, pitch-angle scattering can therefore be decreased by approximately a factor of five, and the parallel mean free path, given (see e.g. Earl 1974) by

$\lambda_{\|}=\frac{3}{v} \kappa_{\|}=\frac{3 v}{8} \int_{-1}^{+1} \mathrm{~d} \mu \frac{\left(1-\mu^{2}\right)^{2}}{D_{\mu \mu}}$

becomes about a factor of 5 larger.

An important quality indeed of two-component turbulence models for reproducing solar-wind observations, is that the parallel mean free path becomes larger by a factor of five. By applying test-particle codes, however, it has been demonstrated (see Shalchi et al. 2004b; Qin et al. 2006) that the assumption $D_{\mu \mu}^{2 \mathrm{D}}=0$ is untrue. According to these numerical studies, there is strong pitch-angle scattering due to two-dimensional modes and, thus, QLT is inappropriate for pitch-angle scattering in the slab/2D composite model. Shalchi et al. (2004b) developed a weakly nonlinear description of pitch-angle and therefore parallel scattering, and perpendicular transport of charged particles. Although, this theory uses some ad hoc assumptions, the weakly 


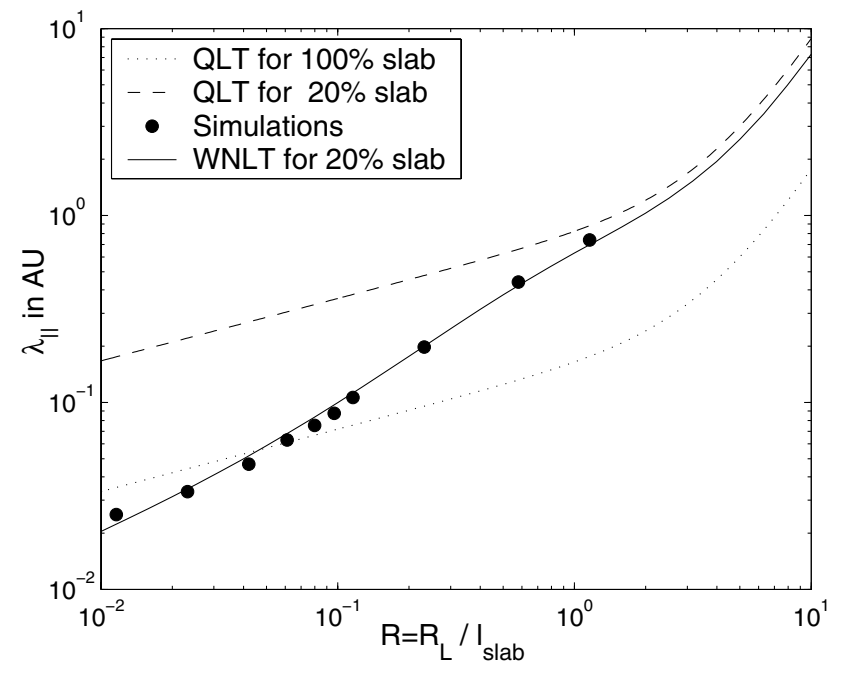

Fig. 1. The parallel mean free path: WNLT results (solid line) in comparison with QLT results (dashed line) and simulations (dots). All results are for $20 \% \mathrm{slab} / 80 \%$ 2D geometry. Also shown is the well-known QLT result for pure slab geometry (dotted line).

nonlinear theory (WNLT) can reproduce test-particle simulations of parallel scattering performed for two-component turbulence (see Fig. 1).

It is the purpose of this article to provide a more systematic description of pitch-angle scattering in pure two-dimensional and two-component turbulence. The main aim is to provide an explanation for QLT is able to reproduce solar-wind observations for parallel mean free paths, but is in disagreement with test-particle simulations.

\section{The correlation tensor of the vector potential in two-dimensional turbulence}

The key input into a transport theory is the correlation tensor of the magnetic fluctuations $P_{l m}(\boldsymbol{k})=\left\langle\delta B_{l}(\boldsymbol{k}) \delta B_{m}^{*}(\boldsymbol{k})\right\rangle$. In the two-dimensional model, the turbulent fields depend only on the perpendicular coordinates $(x, y)$

$\delta B_{i}^{2 \mathrm{D}}(\boldsymbol{x})=\delta B_{i}^{2 \mathrm{D}}(x, y)$

and thus

$P_{l m}(\boldsymbol{k})=A_{z z}(\boldsymbol{k})\left(\delta_{l m} k_{\perp}^{2}-k_{l} k_{m}\right), \quad l, m=x, y$

with

$A_{z z}(\boldsymbol{k}):=g^{2 \mathrm{D}}\left(k_{\perp}\right) \frac{\delta\left(k_{\|}\right)}{k_{\perp}^{3}}$

where we used the two-dimensional (2D) wavespectrum $g^{2 \mathrm{D}}\left(k_{\perp}\right)$. In this model, the wave vectors are aligned perpendicular to the mean field $\boldsymbol{k} \perp \boldsymbol{B}_{0}$, and are, therefore, in a two-dimensional plane. It should be noted, however, that twodimensional models with $\delta B_{z} \neq 0$ can also be formulated. We consider only a two-dimensional model with $\delta B_{z}=0$, which could be called a full two-dimensional model because $\boldsymbol{k} \perp \boldsymbol{B}_{0}$ and $\delta \boldsymbol{B} \perp \boldsymbol{B}_{0}$.

The vector potential $\delta \boldsymbol{A}(\boldsymbol{x})$ is related to the magnetic fields by means of

$\delta \boldsymbol{B}(\boldsymbol{x})=\boldsymbol{\nabla} \times \delta \boldsymbol{A}(\boldsymbol{x})$ and thus

$\delta B_{x}(\boldsymbol{x})=\partial_{y} \delta A_{z}(\boldsymbol{x})-\partial_{z} \delta A_{y}(\boldsymbol{x})=\partial_{y} \delta A_{z}(x, y)$,
$\delta B_{y}(\boldsymbol{x})=\partial_{z} \delta A_{x}(\boldsymbol{x})-\partial_{x} \delta A_{z}(\boldsymbol{x})=-\partial_{x} \delta A_{z}(x, y)$,

where we used $\delta \boldsymbol{A}(\boldsymbol{x})=\delta \boldsymbol{A}(x, y)$ for pure two-dimensional turbulence. In Fourier space, this becomes

$\delta B_{x}\left(k_{x}, k_{y}\right)=\mathrm{i} k_{y} \delta A_{z}\left(k_{x}, k_{y}\right)$

$\delta B_{y}\left(k_{x}, k_{y}\right)=-\mathrm{i} k_{x} \delta A_{z}\left(k_{x}, k_{y}\right)$,

and thus

$$
\begin{aligned}
P_{l m}(\boldsymbol{k}) & =\left\langle\delta B_{l}\left(k_{x}, k_{y}\right) \delta B_{m}^{*}\left(k_{x}, k_{y}\right)\right\rangle \\
& =\left\langle\delta A_{z}\left(k_{x}, k_{y}\right) \delta A_{z}^{*}\left(k_{x}, k_{y}\right)\right\rangle\left[\delta_{l m} k_{\perp}^{2}-k_{l} k_{m}\right] .
\end{aligned}
$$

By comparing Eq. (5) with Eq. (10), the function $A_{z z}(\boldsymbol{k})$ can be related to the correlation function of the vector potential:

$A_{z z}\left(k_{x}, k_{y}\right)=\left\langle\delta A_{z}\left(k_{x}, k_{y}\right) \delta A_{z}^{*}\left(k_{x}, k_{y}\right)\right\rangle$.

In the next section, these results are used to describe cosmic-ray pitch-angle diffusion in pure two-dimensional turbulence.

\section{A general formula for pitch-angle scattering in two-dimensional turbulence}

\subsection{A general relation between pitch-angle and perpendicular transport}

The motion of charged particles in electromagnetic fields is described by the Newton-Lorentz equation

$\frac{\mathrm{d}}{\mathrm{d} t} \boldsymbol{p}=q\left(\boldsymbol{E}+\frac{\boldsymbol{v}}{c} \times \boldsymbol{B}\right)$

Here, the particle charge $q$, the electric and magnetic fields $\boldsymbol{E}$ and $\boldsymbol{B}$, the particle velocity $\boldsymbol{v}$, and the speed of light, $c$, are used. For the mean magnetic field (background field), we assume that

$\langle\boldsymbol{B}\rangle=\boldsymbol{B}_{0}=B_{0} \boldsymbol{e}_{z}$

with the constant background field $B_{0}$. In the case of heliospheric particle propagation, the mean field can be identified with the magnetic field of the Sun. Because of the high conductivity of cosmic plasmas, there are no large-scale electric fields $\langle\boldsymbol{E}\rangle=$ $\boldsymbol{E}_{0}=0$ and we thus have

$\boldsymbol{B}=B_{0} \boldsymbol{e}_{z}+\delta \boldsymbol{B}, \quad \boldsymbol{E}=\delta \boldsymbol{E}$

with the turbulent electric and magnetic fields $(\delta \boldsymbol{E}, \delta \boldsymbol{B})$. In this article, only turbulence models without electric fields $\left(\delta E_{i}=0\right)$ are considered. The main reason for using a model of purely magnetic fluctuations is that the electric fields have not been included in test-particle simulations (e.g. Mace et al. 2000; Qin et al. 2002a,b, 2006). In purely magnetic systems, we have

$\boldsymbol{p} \cdot \dot{\boldsymbol{p}}=q \boldsymbol{p} \cdot \boldsymbol{E}=0$

and thus the kinetic energy of the particle is conserved. Hence, we have $v=$ const., and the parallel component of the equations of motion can be written as:

$\dot{v}_{z}=\Omega\left(v_{x} \frac{\delta B_{y}}{B_{0}}-v_{y} \frac{\delta B_{x}}{B_{0}}\right)$

where we used

$p_{z}=m \frac{v_{z}}{\sqrt{1-v^{2} / c^{2}}}$ 
and the parameter of unperturbed gyrofrequency

$\Omega:=\frac{q B_{0}}{m c} \sqrt{1-v^{2} / c^{2}}$.

By introducing the pitch-angle-cosine $\mu$ of the charged particle

$\mu:=\frac{v_{z}}{v}$

we can derive

$\dot{\mu}=\frac{\Omega}{v B_{0}}\left(v_{x} \delta B_{y}-v_{y} \delta B_{x}\right)$.

With Eq. (8) for pure two-dimensional turbulence we obtain

$\dot{\mu}=-\frac{\Omega}{v B_{0}}\left(v_{x} \partial_{x} \delta A_{z}+v_{y} \partial_{y} \delta A_{z}\right)$.

Because $\delta A_{z}=\delta A_{z}(x, y)$, we have

$\dot{\mu}(t)=-\frac{\Omega}{v B_{0}} \frac{\mathrm{d}}{\mathrm{d} t} \delta A_{z}(x(t), y(t))$

and we finally obtain

$\Delta \mu(t)=-\frac{\Omega}{v B_{0}} \Delta \delta A_{z}(x(t), y(t))$

where we used

$\Delta \mu(t)=\mu(t)-\mu(0)$

$\Delta \delta A_{z}(x(t), y(t))=\delta A_{z}(x(t), y(t))-\delta A_{z}(x(0), y(0))$.

Obviously we can compute the pitch-angle-cosine $\mu(t)$ at time $t$, if we have knowledge of the perpendicular position of the particle $(x(t), y(t))$ at precisely the same time. In the next paragraph, we combine this (exact) relation with a random-phase approximation to deduce a general formula for pitch-angle scattering.

\subsection{Random-phase approximation}

Using a Fourier representation for the vector potential, we can rewrite Eq. (23)

$\Delta \mu(t)=-\frac{\Omega}{v B_{0}} \int \mathrm{d}^{3} k \delta A_{z}(\boldsymbol{k}) \xi(\boldsymbol{k}, t)$

with

$\xi(\boldsymbol{k}, t)=\left[\mathrm{e}^{\mathrm{i} \boldsymbol{k} \cdot \boldsymbol{x}(t)}-\mathrm{e}^{\mathrm{i} \boldsymbol{k} \cdot \boldsymbol{x}(0)}\right]$.

For the mean square displacement in the velocity space

$\sigma_{\mu}^{2}(t):=\left\langle\Delta \mu(t) \Delta \mu^{*}(t)\right\rangle$

we therefore find

$\sigma_{\mu}^{2}(t)=\left(\frac{\Omega}{v B_{0}}\right)^{2} \int \mathrm{d}^{3} k \int \mathrm{d}^{3} k^{\prime}\left\langle\delta A_{z}(\boldsymbol{k}) \delta A_{z}^{*}\left(\boldsymbol{k}^{\prime}\right) \xi(\boldsymbol{k}, t) \xi^{*}\left(\boldsymbol{k}^{\prime}, t\right)\right\rangle$.

To proceed, we have to apply a random-phase approximation (e.g. Corrsin 1959)

$$
\begin{aligned}
& \left\langle\delta A_{z}(\boldsymbol{k}) \delta A_{z}^{*}\left(\boldsymbol{k}^{\prime}\right) \xi(\boldsymbol{k}, t) \xi^{*}\left(\boldsymbol{k}^{\prime}, t\right)\right\rangle= \\
& \left\langle\delta A_{z}(\boldsymbol{k}) \delta A_{z}^{*}\left(\boldsymbol{k}^{\prime}\right)\right\rangle\left\langle\xi(\boldsymbol{k}, t) \xi^{*}\left(\boldsymbol{k}^{\prime}, t\right)\right\rangle .
\end{aligned}
$$

By assuming homogenous turbulence

$\left\langle\delta A_{z}(\boldsymbol{k}) \delta A_{z}^{*}\left(\boldsymbol{k}^{\prime}\right)\right\rangle=\delta\left(\boldsymbol{k}-\boldsymbol{k}^{\prime}\right) A_{z z}(\boldsymbol{k})$ we finally obtain

$\sigma_{\mu}^{2}(t)=\left(\frac{\Omega}{v B_{0}}\right)^{2} \int \mathrm{d}^{3} k A_{z z}(\boldsymbol{k})\left\langle\xi(\boldsymbol{k}, t) \xi^{*}(\boldsymbol{k}, t)\right\rangle$,

with

$\xi(\boldsymbol{k}, t) \xi^{*}(\boldsymbol{k}, t)=2[1-\cos (\boldsymbol{k} \Delta \boldsymbol{x})]$

where we used $\Delta \boldsymbol{x}=\boldsymbol{x}(t)-\boldsymbol{x}(0)$, we obtain

$\sigma_{\mu}^{2}(t)=\left(\frac{\Omega}{v B_{0}}\right)^{2} \int \mathrm{d}^{3} k A_{z z}(\boldsymbol{k})[1-\langle\cos (\boldsymbol{k} \Delta \boldsymbol{x})\rangle]$.

In Eq. (33), we used the correlation tensor of the vector potential $A_{z z}(\boldsymbol{k})$, which we discussed in the last section. This tensor, which is given by Eq. (6), can be combined easily with Eq. (33)

$\sigma_{\mu}^{2}(t)=\left(\frac{\Omega}{v B_{0}}\right)^{2} \int \mathrm{d}^{2} k g^{2 \mathrm{D}}\left(k_{\perp}\right) k_{\perp}^{-3}[1-\langle\cos (\boldsymbol{k} \Delta \boldsymbol{x})\rangle]$

but now with $\boldsymbol{k} \cdot \Delta \boldsymbol{x}=k_{x} \Delta x+k_{y} \Delta y$. To proceed, we have to specify the form of the spectrum $g^{2 \mathrm{D}}\left(k_{\perp}\right)$.

\subsection{Standard spectrum for $g^{2 D}\left(k_{\perp}\right)$}

To describe the two-dimensional wavespectrum, we used a spectrum with energy range $\left(k_{\perp}<l_{2 \mathrm{D}}^{-1}\right)$ and inertial range $\left(k_{\perp}>l_{2 \mathrm{D}}^{-1}\right)$, as proposed by Bieber et al. (1994), but without dissipation range

$g^{2 \mathrm{D}}\left(k_{\perp}\right)=\frac{2 C(v)}{\pi} l_{2 \mathrm{D}} \delta B_{2 \mathrm{D}}^{2}\left(1+k_{\perp}^{2} l_{2 \mathrm{D}}^{2}\right)^{-v}$

where we used the normalization function

$C(v)=\frac{1}{2 \sqrt{\pi}} \frac{\Gamma(v)}{\Gamma(v-1 / 2)}$.

Furthermore, we used the two-dimensional, or perpendicular, bendover scale $l_{2 \mathrm{D}}$, the turbulence energy of the two-dimensional modes $\delta B_{2 \mathrm{D}}^{2}$, and the inertial range spectral-index $2 v$. Therefore, we obtain

$$
\begin{aligned}
\sigma_{\mu}^{2}(t)= & \frac{4}{\pi} C(v) l_{2 \mathrm{D}} \delta B_{2 \mathrm{D}}^{2}\left(\frac{\Omega}{v B_{0}}\right)^{2} \\
& \times \int \mathrm{d}^{2} k \frac{1}{k_{\perp}^{3}}\left(1+k_{\perp}^{2} l_{2 \mathrm{D}}^{2}\right)^{-v}[1-\langle\cos (\boldsymbol{k} \Delta \boldsymbol{x})\rangle] .
\end{aligned}
$$

The only unknown input parameter is the function

$$
\langle\cos (\boldsymbol{k} \Delta \boldsymbol{x}(t))\rangle=\left\langle\cos \left(k_{x} \Delta x(t)+k_{y} \Delta y(t)\right)\right\rangle .
$$

In the next section, we discuss three approaches to approximate this function and the resulting forms of the parameter $\sigma_{\mu}^{2}(t)$.

\section{Modelling perpendicular scattering of the charged particle}

Obviously the perpendicular motion of the particle must be known to describe pitch-angle scattering for pure twodimensional turbulence. In previous theories (see e.g. Shalchi et al. 2004b), we have had to know the particle velocity and the position of the particle, for all timescales. In the approach presented in the last section, we had to know only the function $\langle\cos (\boldsymbol{k} \Delta \boldsymbol{x})\rangle$ in the limit of large timescales. In the following, we consider two approaches for this function. 


\subsection{The quasilinear approach}

Within QLT, we replace the parameter $\Delta \boldsymbol{x}$ in Eq. (37) by the unperturbed orbit:

$$
\begin{aligned}
& \Delta x_{\mathrm{QLT}}(t)=-\frac{v}{\Omega} \sqrt{1-\mu^{2}}\left[\sin \left(\Phi_{0}-\Omega t\right)-\sin \left(\Phi_{0}\right)\right] \\
& \Delta y_{\mathrm{QLT}}(t)=+\frac{v}{\Omega} \sqrt{1-\mu^{2}}\left[\cos \left(\Phi_{0}-\Omega t\right)-\cos \left(\Phi_{0}\right)\right]
\end{aligned}
$$

with the initial Gyrophase $\Phi_{0}$ and the pitch-angle-cosine $\mu$, which is constant in the unperturbed system. We therefore find that the pitch-angle mean square deviation is described by

$$
\begin{aligned}
\sigma_{\mu}^{2}(t)= & \frac{4}{\pi} C(v) l_{2 \mathrm{D}} \delta B_{2 \mathrm{D}}^{2}\left(\frac{\Omega}{v B_{0}}\right)^{2} \\
& \times \int_{0}^{\infty} \frac{\mathrm{d} k_{\perp}}{k_{\perp}^{2}}\left(1+k_{\perp}^{2} l_{2 \mathrm{D}}^{2}\right)^{-v} \\
& \times \int_{0}^{2 \pi} \mathrm{d} \Psi\left[1-\cos \left(k_{\perp} \cos (\Psi) \Delta x_{\mathrm{QLT}}(t)\right.\right. \\
& \left.\left.+k_{\perp} \sin (\Psi) \Delta y_{\mathrm{QLT}}(t)\right)\right]
\end{aligned}
$$

where we used

$k_{x}=k_{\perp} \cos (\Psi)$

$k_{y}=k_{\perp} \sin (\Psi)$.

By combining Eqs. (39) and (40) and by using

$\mathrm{e}^{ \pm \mathrm{i} k \Delta x}=\sum_{n, m} J_{n}(W) J_{m}(W) \mathrm{e}^{ \pm \mathrm{i} n\left(\Psi-\Phi_{0}+\Omega t\right) \mp \mathrm{i} m\left(\Psi-\Phi_{0}\right)}$

and

$$
\int_{0}^{2 \pi} \mathrm{d} \Psi \mathrm{e}^{\mathrm{i}(n-m) \Psi}=2 \pi \delta_{n, m}
$$

we find that

$$
\begin{aligned}
\sigma_{\mu}^{2}(t)= & \frac{8}{\pi} C(v) l_{2 \mathrm{D}} \delta B_{2 \mathrm{D}}^{2}\left(\frac{\Omega}{v B_{0}}\right)^{2} \\
& \times \int_{0}^{\infty} \frac{\mathrm{d} k_{\perp}}{k_{\perp}^{2}}\left(1+k_{\perp}^{2} l_{2 \mathrm{D}}^{2}\right)^{-v} \\
& \times\left[1-\sum_{n=-\infty}^{+\infty} J_{n}^{2}(W) \cos (n \Omega t)\right] .
\end{aligned}
$$

In Eqs. (42)-(44), we used the Bessel functions $J_{n}(x)$ and the parameter $W=k_{\perp} \sqrt{1-\mu^{2}} v / \Omega$. With (see e.g. Abramowitz \& Stegun 1974)

$$
\sum_{n=-\infty}^{\infty} J_{n}^{2}(W)=1
$$

and

$1-\cos (n \Omega t)=2 \sin ^{2}(n \Omega t / 2)$

we obtain

$$
\begin{aligned}
\sigma_{\mu}^{2}(t)= & 16 C(v) l_{2 \mathrm{D}} \delta B_{2 \mathrm{D}}^{2}\left(\frac{\Omega}{v B_{0}}\right)^{2} \\
& \times \int_{0}^{\infty} \frac{\mathrm{d} k_{\perp}}{k_{\perp}^{2}}\left(1+k_{\perp}^{2} l_{2 \mathrm{D}}^{2}\right)^{-v} \\
& \times \sum_{n=-\infty}^{\infty} J_{n}^{2}(W) \sin ^{2}(n \Omega t / 2) .
\end{aligned}
$$

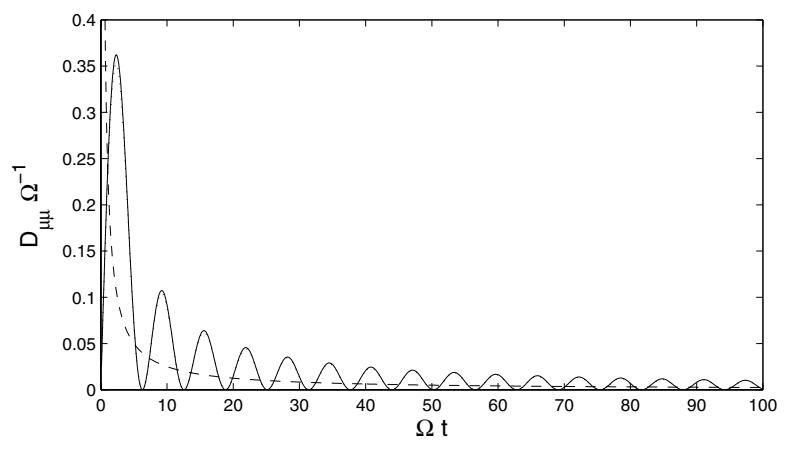

Fig. 2. The quasilinear pitch-angle Fokker-Planck coefficient $D_{\mu \mu} \Omega^{-1}$ as a function of the dimensionless time $\Omega t$ for pure two-dimensional turbulence. We show the analytical result (dotted line), the averaged result (dashed line), and the numerical result (solid line) for $R_{\mathrm{L}}=0.01 l_{2 \mathrm{D}}$. We note that the analytical result and the numerical result are nearly identical.

In Appendix A, we demonstrate that for $R_{\mathrm{L}} \sqrt{1-\mu^{2}} \ll l_{2 \mathrm{D}}$ (low energetic particles), we have

$\sigma_{\mu}^{2}(t)=2\left(1-\mu^{2}\right)\left(\frac{\delta B_{2 \mathrm{D}}}{B_{0}}\right)^{2} \sin ^{2}\left(\frac{\Omega t}{2}\right)$.

By defining a time-dependent pitch-angle Fokker-Planck coefficient as

$D_{\mu \mu}(t):=\frac{\sigma_{\mu}^{2}(t)}{2 t} \equiv \frac{\left\langle\Delta \mu \Delta \mu^{*}\right\rangle}{2 t}$

we find for pure two-dimensional turbulence within QLT

$D_{\mu \mu}^{2 \mathrm{D}, \mathrm{QLT}}(t)=\left(1-\mu^{2}\right)\left(\frac{\delta B_{2 \mathrm{D}}}{B_{0}}\right)^{2} \sin ^{2}\left(\frac{\Omega t}{2}\right) t^{-1}$.

Alternatively, one can consider the time average of Eq. (48)

$\bar{\sigma}_{\mu}^{2}(t)=\left(1-\mu^{2}\right)\left(\frac{\delta B_{2 D}}{B_{0}}\right)^{2}$

and therefore

$\bar{D}_{\mu \mu}^{2 \mathrm{D}, \mathrm{QLT}}(t)=\frac{1}{2 t}\left(1-\mu^{2}\right)\left(\frac{\delta B_{2 \mathrm{D}}}{B_{0}}\right)^{2}$.

The following results are illustrated in Fig. 2: the analytical result of Eq. (50), the averaged result of Eq. (52), and the result obtained by solving the series and the integral of Eq. (47) numerically.

According to Eq. (50), we have

$D_{\mu \mu}^{2 \mathrm{D}, \mathrm{QLT}}(t) \sim\left\{\begin{array}{cl}0 & \text { if } \Omega t=2 \pi m \\ t^{-1} & \text { if } \Omega t \neq 2 \pi m\end{array}\right.$

where $m$ is an integer number. In Fig. 2, we show the periodic behaviour of $D_{\mu \mu}$. After a gyroperiod, we have $\Delta A=0$ and thus $D_{\mu \mu}=0$ (see also Eq. (23)). Only during a period in which the orbit is not closed we do find nonvanishing pitch-angle scattering within QLT. Obviously we find

$D_{\mu \mu}^{2 \mathrm{D}, \mathrm{QLT}}=D_{\mu \mu}^{2 \mathrm{D}, \mathrm{QLT}}(t \rightarrow \infty) \rightarrow 0$

within QLT and two-dimensional turbulence. This result was reported previously by Qin et al. (2006) using a different, simpler approach. Thus, Eqs. (1) and (2) are valid within quasilinear theory. 


\subsection{Stochastic Gaussian perpendicular motion}

We assume that the particle position can be approximated by the guiding-center motion. In combination with the assumption that these are distributed in a similar way as Gaussian functions, we have for axisymmetric turbulence

$\langle\cos (\boldsymbol{k} \Delta \boldsymbol{x})\rangle=\mathrm{e}^{-\frac{1}{2} \sigma_{\perp}^{2}(t) k_{\perp}^{2}}$

where we used the time-dependent mean square deviation $\sigma_{\perp}^{2}(t)=\left\langle(\Delta x(t))^{2}\right\rangle=\left\langle(\Delta y(t))^{2}\right\rangle$ in the perpendicular direction. For diffusive behaviour, for instance, we would have $\sigma_{\perp}^{2}=$ $2 t D_{\perp}$ with the perpendicular Fokker-Planck coefficient $D_{\perp}$. Equation (37) with Eq. (55) gives

$$
\begin{aligned}
\sigma_{\mu}^{2}(t)= & \frac{4}{\pi} C(v) l_{2 \mathrm{D}} \delta B_{2 \mathrm{D}}^{2}\left(\frac{\Omega}{v B_{0}}\right)^{2} \\
& \times \int \mathrm{d}^{2} k \frac{1}{k_{\perp}^{3}}\left(1+k_{\perp}^{2} l_{2 \mathrm{D}}^{2}\right)^{-v}\left[1-\mathrm{e}^{-\frac{1}{2} \sigma_{\perp}^{2}(t) k_{\perp}^{2}}\right] .
\end{aligned}
$$

The remaining integral is investigated in Appendix B, where we demonstrate that we obtain two different solutions, namely

$\sigma_{\mu}^{2}(t) \approx\left\{\begin{array}{cl}\left(\frac{\delta B_{2 \mathrm{D}}}{B_{0}}\right)^{2} \frac{\sigma_{\perp}^{2}(t)}{R_{\mathrm{L}}^{2}} & \text { for } \sigma_{\perp}^{2}(t) \ll l_{2 \mathrm{D}}^{2} \\ 8 \sqrt{\frac{\pi}{2}} C(v)\left(\frac{\delta B_{2 \mathrm{D}}}{B_{0}}\right)^{2} \frac{l_{2 \mathrm{D}} \sqrt{\sigma_{\perp}^{2}(t)}}{R_{\mathrm{L}}^{2}} & \text { for } \sigma_{\perp}^{2}(t) \gg l_{2 \mathrm{D}}^{2}\end{array}\right.$

with the Larmor-radius $R_{\mathrm{L}}=v / \Omega$.

We are interested mainly in the late time limit $(t \rightarrow \infty)$. In this case, we expect $\sigma_{\perp}^{2} \rightarrow \infty$. Hence, the second case is much more interesting and important. For the time-dependent pitchangle Fokker-Planck coefficient (Eq. (49)), we find

$D_{\mu \mu}^{2 \mathrm{D}, \mathrm{NL}}(t)=\frac{4}{t} \sqrt{\pi} C(v) l_{2 \mathrm{D}}^{2} \delta B_{2 \mathrm{D}}^{2}\left(\frac{\Omega}{v B_{0}}\right)^{2} \sqrt{\frac{\sigma_{\perp}^{2}(t)}{2 l_{2 \mathrm{D}}^{2}}}$.

We define the time-dependent perpendicular Fokker-Planck coefficient to be

$D_{\perp}(t):=\frac{\sigma_{\perp}^{2}(t)}{2 t}$

to find

$D_{\mu \mu}^{2 \mathrm{D}, \mathrm{NL}}(t)=4 C(v) l_{2 \mathrm{D}} \delta B_{2 \mathrm{D}}^{2}\left(\frac{\Omega}{v B_{0}}\right)^{2} \sqrt{\frac{\pi D_{\perp}(t)}{t}}$.

This result cannot be used to determine a diffusion coefficent without knowing the other coefficient. Therefore we must consider certain assumptions for $D_{\perp}(t)$. In cases where perpendicular transport behaves diffusively, that is $D_{\perp}(t)=D_{\perp}=$ const., we have $D_{\mu \mu}(t) \sim t^{-1 / 2}$ and find a subdiffusive behaviour of pitch-angle scattering. If we assume a subdiffusive behaviour of perpendicular transport, as in the slab model $\left(D_{\perp}(t) \sim t^{-1 / 2}\right)$, we find a strong subdiffusive behaviour of pitch-angle scattering $\left(D_{\mu \mu}(t) \sim t^{-3 / 4}\right)$. It appears that if we assume that perpendicular transport is diffusive or subdiffusive, we find a subdiffusive behaviour of pitch-angle transport. Diffusive pitch-angle scattering can only be obtained for $D_{\perp}(t) \sim t$, which corresponds to ballistic perpendicular motion.

\subsection{Alternative approach for $v=1$}

According to the results derived in the previous paragraphs, the inertial range spectral-index $2 v$ does not have an influence on the late time behaviour of pitch-angle scattering (see e.g. Eq. (60)). It is the purpose of the following calculations to derive a general formula for pitch-angle scattering by setting $v=1$. In this case, Eq. (37) can be written as

$\sigma_{\mu}^{2}(t)=\frac{2}{\pi^{2}}\left(\frac{l_{2 \mathrm{D}}}{R_{\mathrm{L}}}\right)^{2}\left(\frac{\delta B_{2 \mathrm{D}}^{2}}{B_{0}}\right)^{2}\langle J(t)\rangle$

Here we used $C(v=1)=(2 \pi)^{-1}$ and

$J(t)=\int_{0}^{2 \pi} \mathrm{d} \Psi I(t, \Psi)$

with

$I(t, \Psi)=\int_{0}^{\infty} \mathrm{d} x \frac{1-\cos (\rho x)}{x^{2}+x^{4}}$

In Eq. (63), we introduced the parameter

$\rho=\left|\frac{\Delta x(t)}{l_{2 \mathrm{D}}} \cos \Psi+\frac{\Delta y(t)}{l_{2 \mathrm{D}}} \sin \Psi\right|$.

The integral of Eq. (63) can be solved (see e.g. Gradshteyn \& Ryzhik 2000)

$I(t, \Psi)=\frac{\pi}{2}\left(\rho-1+\mathrm{e}^{-\rho}\right)$

and Eq. (62) becomes

$J(t)=\frac{\pi}{2} \int_{0}^{2 \pi} \mathrm{d} \Psi\left(\rho-1+\mathrm{e}^{-\rho}\right)$.

To proceed, we write

$\Delta x(t)=r(t) \cos \phi(t)$

$\Delta y(t)=r(t) \sin \phi(t)$

and thus we have

$\rho=\frac{r(t)}{l_{2 \mathrm{D}}}|\cos (\Psi-\phi(t))|$.

The integral of Eq. (66) can be expressed by modified Bessel functions $I_{v}(z)$ and modified Struve functions $L_{v}(z)$ (see Abramowitz \& Stegun 1974; Gradshteyn \& Ryzhik 2000)

$J(t)=\pi^{2}\left[\frac{2}{\pi} \frac{r(t)}{l_{2 \mathrm{D}}}-1+I_{0}\left(\frac{r(t)}{l_{2 \mathrm{D}}}\right)-L_{0}\left(\frac{r(t)}{l_{2 \mathrm{D}}}\right)\right]$.

Finally we find for pitch-angle scattering, Eq. (69) combined with Eq. (61), that

$$
\begin{aligned}
\sigma_{\mu}^{2}(t)= & 2\left(\frac{l_{2 \mathrm{D}}}{R_{\mathrm{L}}}\right)^{2}\left(\frac{\delta B_{2 \mathrm{D}}}{B_{0}}\right)^{2} \\
& \times\left\langle\frac{2}{\pi} \frac{r(t)}{l_{2 \mathrm{D}}}-1+I_{0}\left(\frac{r(t)}{l_{2 \mathrm{D}}}\right)-L_{0}\left(\frac{r(t)}{l_{2 \mathrm{D}}}\right)\right\rangle .
\end{aligned}
$$

In Appendix C, we consider two extreme cases, namely $r(t) \ll$ $l_{2 \mathrm{D}}$ and $r(t) \gg l_{2 \mathrm{D}}$. We derive that

$\sigma_{\mu}^{2}(t) \approx\left\{\begin{array}{cl}\frac{1}{2}\left(\frac{\delta B_{2 \mathrm{D}}}{B_{0}}\right)^{2} \frac{\left\langle r^{2}(t)\right\rangle}{R_{\mathrm{L}}^{2}} & \text { for } r(t) \ll l_{2 \mathrm{D}} \\ \frac{4}{\pi}\left(\frac{l_{2 \mathrm{D}}}{R_{\mathrm{L}}}\right)^{2}\left(\frac{\delta B_{2 \mathrm{D}}}{B_{0}}\right)^{2} \frac{\langle r(t)\rangle}{l_{2 \mathrm{D}}} & \text { for } r(t) \gg l_{2 \mathrm{D}} .\end{array}\right.$ 
The first limit corresponds to the case in which the particle has not moved more than a bendover scale $l_{2 \mathrm{D}}$. In cases where the perpendicular motion is a stochastic motion, one could say that the propability to find the particle at $r(t) \ll l_{2 \mathrm{D}}$ is very low. In the following, we consider the two examples of the previous paragraphs and we demonstrate that these results can be derived directly from Eq. (71). Furthermore, we employ a non-Gaussian model to explore the influence of the distribution function.

\subsubsection{Recovery of the QLT result for low rigidites}

Within QLT, we have, using Eq. (39),

$$
\begin{aligned}
r_{\mathrm{QLT}}(t) & =\sqrt{(\Delta x(t))^{2}+(\Delta y(t))^{2}} \\
& =2 R_{\mathrm{L}} \sqrt{1-\mu^{2}}\left|\sin \left(\frac{\Omega t}{2}\right)\right|
\end{aligned}
$$

where we have set $\Phi_{0}=0$. For low energetic particles, we have $R_{\mathrm{L}} \ll l_{2 \mathrm{D}}$ and thus $r_{\mathrm{QLT}}(t) \ll l_{2 \mathrm{D}}$. Therefore the first case of Eq. (71) has to be applied to find

$\sigma_{\mu}^{2}(t) \approx 2\left(1-\mu^{2}\right)\left(\frac{\delta B_{2 \mathrm{D}}}{B_{0}}\right)^{2} \sin ^{2}\left(\frac{\Omega t}{2}\right)$,

which is in precise agreement with the result derived previously in Eq. (48).

\subsubsection{Recovery of the result for the Gaussian motion}

We now assume a stochastic motion of the particle. Furthermore, we are interested in the case that the probability to find the particle at $r(t) \gg l_{2 \mathrm{D}}$ is high. In this case, we have to apply the second formula of Eq. (71), which provides $\sigma_{\mu}^{2}(t) \sim\langle r(t)\rangle$. By assuming a Gaussian distribution

$f_{\mathrm{G}}(r, t)=\frac{1}{2 \pi \sigma_{\perp}^{2}} \mathrm{e}^{-\frac{r^{2}}{2 \sigma_{\perp}^{2}(t)}}$

in the perpendicular direction, we have

$$
\begin{aligned}
\langle r(t)\rangle_{\mathrm{G}} & =\frac{1}{\left\langle(\Delta x(t))^{2}\right\rangle} \int_{0}^{\infty} \mathrm{d} r r^{2} \mathrm{e}^{-\frac{r^{2}}{2 \sigma_{\perp}^{2}(t)}} \\
& =\sqrt{\frac{\pi}{2}} \sqrt{\sigma_{\perp}^{2}(t)}
\end{aligned}
$$

and with Eq. (71), we find

$\sigma_{\mu}^{2}(t) \approx \frac{4}{\sqrt{2 \pi}}\left(\frac{l_{2 \mathrm{D}}}{R_{\mathrm{L}}}\right)^{2}\left(\frac{\delta B_{2 \mathrm{D}}}{B_{0}}\right)^{2} \frac{\sqrt{\sigma_{\perp}^{2}}}{l_{2 \mathrm{D}}}$

which agrees with Eq. (57) for $v=1$. Obviously we can derive the quasilinear limit as well as the stochastic Gaussian limit from Eq. (70). However, Eq. (71) is much more general (requiring that $v=1$ is a good approximation) and can also be evaluated for a non-Gaussian stochastic motion of the charged particles.

\subsubsection{The result for a non-Gaussian motion}

We continue to assume a stochastic motion of the charged particles. However, instead of assuming a Gaussian distribution, we employ a constant probability function

$f(r, t)=\left\{\begin{array}{c}f_{0} \text { for } r \leq r_{0} \\ 0 \text { for } r>r_{0} .\end{array}\right.$
To employ this ansatz for the distribution function, we have to calculate the moments:

$$
\begin{gathered}
1=2 \pi \int_{0}^{\infty} \mathrm{d} r r f(r, t)=\pi f_{0} r_{0}^{2} \\
\langle r\rangle=2 \pi \int_{0}^{\infty} \mathrm{d} r r^{2} f(r, t)=2 \pi f_{0} \frac{r_{0}^{3}}{3} \\
\left\langle r^{2}\right\rangle=2 \pi \int_{0}^{\infty} \mathrm{d} r r^{3} f(r, t)=2 \pi f_{0} \frac{r_{0}^{4}}{4} .
\end{gathered}
$$

By assembling these results, we can deduce

$$
\begin{aligned}
\langle r\rangle & =\frac{2}{3} r_{0} \\
\left\langle r^{2}\right\rangle & =\frac{1}{2} r_{0}^{2}
\end{aligned}
$$

and therefore

$\langle r\rangle=\frac{2}{3} \sqrt{2\left\langle r^{2}\right\rangle}$.

By combining these results with the axisymmetric assumption $\left\langle r^{2}\right\rangle=\left\langle(\Delta x)^{2}\right\rangle+\left\langle(\Delta y)^{2}\right\rangle=2 \sigma_{\perp}^{2}$, it follows that

$\langle r\rangle=\frac{2}{3} \sqrt{2 \sigma_{\perp}^{2}}$,

which implies a similar dependence on the parameter $\sigma_{\perp}^{2}$ as found for the Gaussian distribution in Eq. (75). It appears that the form of the particle distribution function has only a weak influence on the late time behaviour. In both cases, we find $\sigma_{\mu}^{2} \sim \sqrt{\sigma_{\perp}^{2}}$.

\section{Different forms of the wavespectrum in the energy range}

The results presented in the previous section are valid for the wave spectrum of Eq. (35). However, it can be seen easily, for example in the calculations presented in Appendix B, that the energy range, corresponding to the part of the spectrum with $k_{\perp} \leq l_{2 \mathrm{D}}^{-1}$, controls the relation between pitch-angle scattering and perpendicular scattering.

We explore the influence of the energy range by adopting the following form

$$
\begin{aligned}
g^{2 \mathrm{D}}\left(k_{\perp}\right)= & \frac{d_{1}}{2 \pi} l_{2 \mathrm{D}} \delta B_{2 \mathrm{D}}^{2} \\
& \times\left\{\begin{array}{ccc}
0 & \text { if } & k_{\perp}<k_{\min } \\
\left(k_{\perp} l_{2 \mathrm{D}}\right)^{-q} & \text { if } & k_{\min } \leq k_{\perp} \leq l_{2 \mathrm{D}}^{-1} \\
\left(k_{\perp} l_{2 \mathrm{D}}\right)^{-2 v} & \text { if } & l_{2 \mathrm{D}}^{-1}<k_{\perp} .
\end{array}\right.
\end{aligned}
$$

In comparison to the standard spectrum in Eq. (35), we introduced two additional parameters, namely the cut-off wavenumber $k_{\min }$ and the energy range spectral-index $q$. The spectrum is correctly normalized for $q<1, x_{\min }=k_{\min } l_{2 \mathrm{D}} \ll 1$, and

$d_{1}=\left(\frac{1}{1-q}+\frac{1}{2 v-1}\right)^{-1}$.

For the stochastic Gaussian model, Eq. (33) then becomes

$$
\begin{aligned}
\sigma_{\mu}^{2}= & d_{1}\left(\frac{\Omega}{v B_{0}}\right)^{2} l_{2 \mathrm{D}} \delta B_{2 \mathrm{D}}^{2} \\
& \times\left[\int_{k_{\min }}^{l_{2 \mathrm{D}}^{-1}} \mathrm{~d} k_{\perp}\left(k_{\perp} l_{2 \mathrm{D}}\right)^{-q}\left(1-\mathrm{e}^{-\sigma_{\perp}^{2} k_{\perp}^{2} / 2}\right)\right. \\
& \left.+\int_{l_{2 \mathrm{D}}^{-1}}^{\infty} \mathrm{d} k_{\perp}\left(k_{\perp} l_{2 \mathrm{D}}\right)^{-2 v}\left(1-\mathrm{e}^{-\sigma_{\perp}^{2} k_{\perp}^{2} / 2}\right)\right] .
\end{aligned}
$$


In Appendix D, we evaluate this formula. There, we derive the relation

$\sigma_{\mu}^{2}(t)=\frac{d_{1}}{q+1} \frac{l_{2 \mathrm{D}}^{2}}{R_{\mathrm{L}}^{2}} \frac{\delta B_{2 \mathrm{D}}^{2}}{B_{0}^{2}} \Gamma\left(\frac{1-q}{2}\right)\left(\frac{\sigma_{\perp}^{2}}{2 l_{2 \mathrm{D}}^{2}}\right)^{(q+1) / 2}$

and therefore

$\sigma_{\mu}^{2}(t) \sim\left(\sigma_{\perp}^{2}\right)^{(q+1) / 2}$.

Obviously the relation $\sigma_{\mu}^{2}(t) \sim \sigma_{\perp}^{2}$ can be obtained only for $q \rightarrow 1$; only if this relation is valid, we can have a diffusive behaviour of perpendicular and pitch-angle scattering simultaneously. We find a strong subdiffusive behaviour of pitch-angle scattering in all other cases in particular for negative $q$, which corresponds to an increasing spectrum in the energy range, as proposed by Matthaeus et al. (2007).

\section{Results for the slab/2D composite model}

We have so far employed a turbulence model with only 2D fluctuations $\left(\delta B_{i}(\boldsymbol{x})=\delta B_{i}(x, y)\right)$. A full three-dimensional model for which the turbulent magnetic-field vector depends on all three spatial coordinates, is however more realistic. A simple model to approximate real turbulence is the so-called slab/2D model where we assume a superposition of pure slab and pure 2D fluctuations. This model can be justified by using at least four arguments:

1. Due to the separation of these two one- and two-dimensional contributions, the two-component model is tractable from a mathematical point of view. Full analytical calculations are possible for all spectra used in this paper and simple analytical formulas can be derived. These simple formulas are useful for applications such as solar-wind modulation studies.

2. Solar-wind observations for magnetic self-correlations (see Matthaeus et al. 1990) have the form of a so-called maltesecross, suggesting that the two-component model is a good approximation for solar-wind turbulence.

3. In Bieber et al. (1994) and Shalchi et al. (2006), it was demonstrated that the two-component model can be used in cosmic-ray scattering theory for reproducing observed particle mean free paths in the solar-wind. We can therefore conclude that the slab/2D model is sufficient accurate for applications in cosmic-ray scattering theory.

4. Magnetohydrodynamic turbulence was investigated numerically using incompressible 2D (Shebalin et al. 1983; Grappin 1986) and later in 3D (Carbone \& Veltri 1990; Oughton et al. 1994; Matthaeus et al. 1996) simulations, with consistent results. The basis conclusion is that spectral transfer of energy proceeds more rapidly in wavevectors perpendicular to the mean magnetic field $\boldsymbol{B}_{0}$ (for a review see Horbury et al. 2005).

Obviously there are strong indications from measurements, numerical simulations of turbulence, and applications in cosmicray scattering theory that the two-component model is a good approximation. For completeness, however, it should be mentioned that other turbulence models are available which go beyond the two-component model (see e.g., Goldreich \& Sridhar 1995; Galtier et al. 2002; Cho et al. 2002; Cho \& Lazarian 2002, 2006). To our knowledge, however, it has never been demonstrated that these improvements of turbulence models are important for applications in cosmic-ray transport theory. Since most cosmic-ray scattering studies have focused on transport in the solar-wind, and due to its better analytical tractability, we employ the slab/2D composite model in the current section. In this case, the pitch-angle Fokker-Planck coefficient can be written as a superposition of a slab and a two-dimensional coefficient: see Eq. (1).

\subsection{General results for the slab/2D model}

For the parameter $D_{\mu \mu}^{2 \mathrm{D}}(t)$, we can use the results of the last section and for $D_{\mu \mu}^{\text {slab }}$ we employ the quasilinear result. For the magnetostatic slab model and the spectrum

$g^{\text {slab }}\left(k_{\|}\right)=\frac{C(v)}{2 \pi} l_{\text {slab }} \delta B_{\text {slab }}^{2}\left(1+k_{\|}^{2} l_{\text {slab }}^{2}\right)^{-v}$,

we find according to Shalchi et al. (2004a, Appendix A) that

$$
\begin{aligned}
D_{\mu \mu}^{\text {slab,QLT }}= & \frac{\pi C(v) v}{l_{\text {slab }}}\left(\frac{\delta B_{\text {slab }}}{B_{0}}\right)^{2} \\
& \times\left(1-\mu^{2}\right) \mu^{2 v-1} R^{2 v-2}\left(1+\mu^{2} R^{2}\right)^{-v}
\end{aligned}
$$

where we used the dimensionless rigidity $R=R_{\mathrm{L}} / l_{\text {slab }}=$ $v /\left(\Omega l_{\text {slab }}\right)$. In the last two equations, we have used the slab bendover scale $l_{\text {slab }}$, the strength of the slab magnetic field $\delta B_{\text {slab }}^{2}$, and the inertial-range spectral-index $2 v$. To proceed, it is convenient to calculate the dimensionless pitch-angle Fokker-Planck coefficient

$\tilde{D}_{\mu \mu}:=D_{\mu \mu} \frac{l_{\text {slab }}}{v}$

and thus we find

$$
\begin{aligned}
\tilde{D}_{\mu \mu}^{\text {slab }, \mathrm{QLT}}= & \pi C(v)\left(\frac{\delta B_{\text {slab }}}{B_{0}}\right)^{2} \\
& \times\left(1-\mu^{2}\right) \mu^{2 v-1} R^{2 v-2}\left(1+\mu^{2} R^{2}\right)^{-v}
\end{aligned}
$$

For the two-dimensional coefficient, we employ the stochastic Gaussian model from Eq. (60), which can be written as

$\tilde{D}_{\mu \mu}^{2 \mathrm{D}, \mathrm{NL}}(t)=4 C(v) \frac{l_{2 \mathrm{D}}}{l_{\text {slab }}}\left(\frac{\delta B_{2 \mathrm{D}}}{B_{0}}\right)^{2} R^{-2} \sqrt{\frac{\pi D_{\perp}(t)}{v^{2} t}}$

By using the (dimensionless) parameters

$$
\begin{aligned}
\tau & =\frac{v t}{l_{\text {slab }}} \\
\tilde{D}_{\perp} & =\frac{D_{\perp}}{v l_{\text {slab }}}
\end{aligned}
$$

we finally find

$\tilde{D}_{\mu \mu}^{2 \mathrm{D}, \mathrm{NL}}(t)=4 C(v) \frac{l_{2 \mathrm{D}}}{l_{\text {slab }}}\left(\frac{\delta B_{2 \mathrm{D}}}{B_{0}}\right)^{2} R^{-2} \sqrt{\frac{\pi \tilde{D}_{\perp}(\tau)}{\tau}}$.

Thus, the total pitch-angle Fokker-Planck coefficient has the following time-dependence

$\tilde{D}_{\mu \mu}^{\text {Comp }}(\tau) \sim\left[1+\right.$ const. $\left.\sqrt{\frac{\tilde{D}_{\perp}(\tau)}{\tau}}\right]$.

In the limit of infinitely large timescales $(\tau \rightarrow \infty)$, the total pitch-angle Fokker-Planck coefficient approaches the (diffusive) slab-limit. 


\subsection{Results for turbulence parameters at 1 AU heliocentric distance}

We employ the following parameter set, which should be appropriate for the solar-wind at $1 \mathrm{AU}$ heliocentric distance:

$$
\begin{aligned}
\frac{\delta B_{\text {slab }}^{2}}{B_{0}^{2}} & =0.2 \\
\frac{\delta B_{2 \mathrm{D}}^{2}}{B_{0}^{2}} & =0.8 \\
v & =5 / 6 \Rightarrow C(v)=0.1189 \\
l_{2 \mathrm{D}} / l_{\text {slab }} & =0.1 .
\end{aligned}
$$

Furthermore, for the sake of comparison, we consider $\mu^{2}=1 / 2$ and replace

$\tilde{D}_{\perp}(\tau) \rightarrow \frac{\kappa_{\perp}(\tau)}{v l_{\text {slab }}}=\frac{\lambda_{\perp}(\tau)}{3 l_{\text {slab }}}$

to find

$$
\begin{aligned}
\tilde{D}_{\mu \mu}^{\text {slab,QLT }} & \approx 0.03 R^{-1 / 3}\left(1-\frac{1}{2} R^{2}\right)^{-5 / 6} \\
\tilde{D}_{\mu \mu}^{2 \mathrm{D}, \mathrm{NL}}(\tau) & \approx 0.04 R^{-2} \sqrt{\frac{\lambda_{\perp}(\tau)}{\tau l_{\text {slab }}}} .
\end{aligned}
$$

In Eq. (96), we used the (pitch-angle independent) perpendicular spatial diffusion coefficient $\kappa_{\perp}$ and the perpendicular mean free path $\lambda_{\perp}$. For $R \ll 1$, we find for two-component turbulence

$\tilde{D}_{\mu \mu}^{\text {Comp }}(\tau) \approx 0.03 R^{-1 / 3}\left[1+\frac{4}{3} R^{-5 / 3} \sqrt{\frac{\lambda_{\perp}(\tau)}{\tau l_{\text {slab }}}}\right]$.

Obviously, for low cosmic-ray rigidities we have to consider very late times to find a negligible small two-dimensional contribution. In Fig. 3, we showed the pitch-angle Fokker-Planck coefficient for two different values of the dimensionless rigidity $R$, by applying Eq. (98). As a first example, we assume $R=1$, which corresponds to higher rigidities and therefore to cosmic particles with higher energy. In this case, we find for early times that the numerical result is equal to the analytical slab result, which corresponds to QLT. It appears that the nonlinear effect, which is basically the difference between the two lower lines in Fig. 3, is negligible small. As a second example, we show results for lower rigidities $(R=0.1)$. In this case, we find for early times a large difference between the nonlinear result and QLT: see Fig. 3. For later times, however, the nonlinear curve approaches the QLT results asymptotically. In the limit $t \rightarrow \infty$, we find $D_{\mu \mu}(\tau) \rightarrow D_{\mu \mu}^{\mathrm{QLT}}$. Obviously QLT is correct for pitch-angle scattering of high energetic particles with $R \geq 1$. This statement is in agreement with previous results (see e.g., Fig. 1). For lower particle energies, we clearly find nonvanishing nonlinear effects for earlier times, in agreement with previous articles (see again Fig. 1). New, however, is that this nonlinear effect damps out for very late times. For short time scales, which are usually considered in test-particle simulations, (see next section), we therefore expect to see a strong nonlinear effect for low energetic particles. For cases where we have long time scales (e.g. real particles), QLT could be valid. This conclusion provides a natural explanation why QLT is able to reproduce solar-wind observations as demonstrated in previous articles (e.g. Bieber et al. 1994; Shalchi et al. 2006) but unable to reproduce simulations (see Fig. 1).

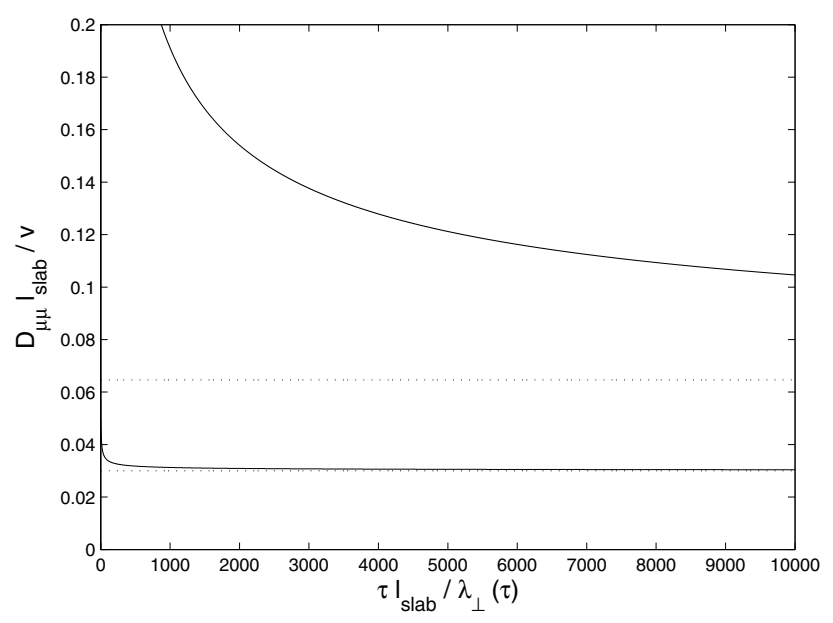

Fig. 3. The dimensionless pitch-angle Fokker-Planck coefficient $\tilde{D}_{\mu \mu}$ as a function of dimensionless time $\tau$. We shown the quasilinear slab limits (dotted lines) and the analytical results for slab/2D composite geometry (solid line). We have shown the results for two values of the dimensionless rigidity, namely for $R=1$ (lower graphs) and $R=0.1$ (upper graphs). For lower rigidity, it needs a much longer time before the result reaches the quasilinear slab limit.

Besides the question of whether nonlinear effects are important for pitch-angle scattering or not, we learn from these new results that there is another interesting feature. For earlier times, pitch-angle scattering in two-component turbulence behaves subdiffusively, in the nonlinear regime. For later times, however, we detect a change to the diffusive behavior predicted by QLT. It appears that the characteristics of pitch-angle scattering varies with time.

\section{Test-particle simulations}

Some assumptions were used to obtain the results of the last section; those were

- The Corrsin (or random phase) approximation is valid.

- The gyromotion of the particle can be neglected for late times.

- The guiding center distribution function can be approximated by a Gaussian function.

Because these assumption are uncertain, it is essential to test our results by comparing them with test-particle simulations. Equation (3) relates the pitch-angle Fokker-Planck coefficient to the cosmic-ray parallel mean free path. However, this formula is valid only for a diffusive behaviour of pitch-angle scattering and can, therefore, not be applied to the subdiffusive results derived in this article. It will be the subject of future work to derive a replacement for Eq. (3), that can also be applied for non-diffusive pitch-angle scattering. However, one would expect that a subdiffusive behaviour of pitch-angle scattering leads to a superdiffusive parallel motion of the particles. Thus, we have to reinvestigate previous test-particle simulations to find out whether parallel scattering is diffusive or not.

By assuming the form $\tilde{\kappa}_{i}(t)=a_{i} \tau^{b_{i}}$, where we use $i=\perp$ for perpendicular scattering and $i=\|$ for parallel scattering, of the diffusion coefficients, we deduce the time-dependence from numerical data by using $b_{i}=\left(\ln \tilde{\kappa}_{i}(\tau)-\ln a_{i}\right) / \ln \tau \approx\left(\ln \tilde{\kappa}_{i}(\tau)\right) / \ln \tau$ in the late-time limit, where $\tilde{\kappa}_{i}$ denotes the dimensionless diffusion coefficients obtained by the simulations. The exponents 


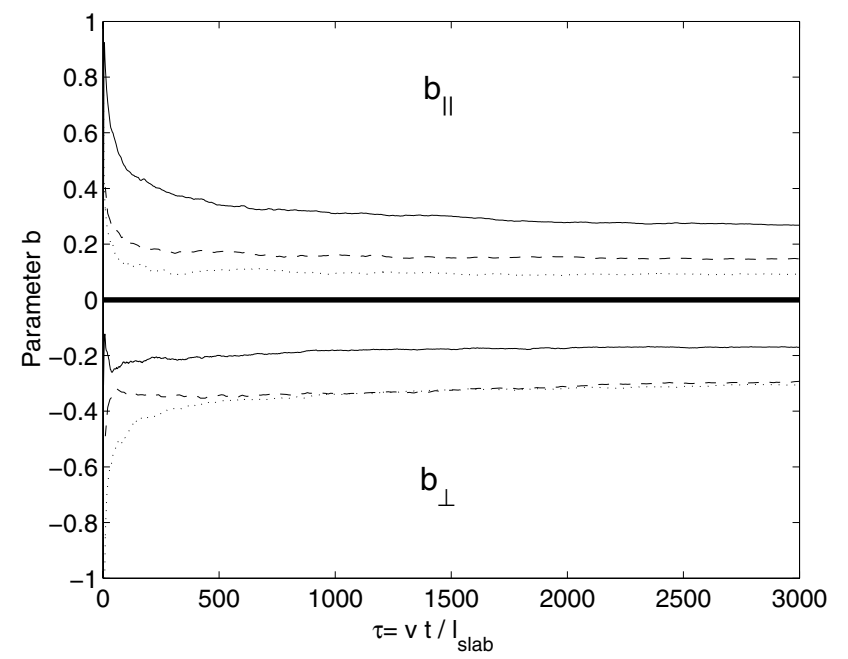

Fig. 4. The parameters $b_{\|}$and $b_{\perp}$ as a function of time for different values of the dimensionless rigidity: $R=10^{-3}$ (dotted line), $R=10^{-2}$ (dashed line), and $R=10^{-1}$ (solid line). Clearly we find a weakly superdiffusive behavior of parallel transport $\left(b_{\|}>0\right)$ and a weakly subdiffusive behavior of perpendicular transport $\left(b_{\perp}<0\right)$.

for the parallel $b_{\|}$and perpendicular $b_{\perp}$ diffusion coefficients are depicted in Fig. 4 for different values of the dimensionless rigidity $R=R_{\mathrm{L}} / l_{\text {slab }}$. As shown, the test-particle code provides a weakly superdiffusive behaviour of parallel transport, in addition to a weakly subdiffusive behavior of perpendicular transport. It seems that a superdiffusive behaviour as predicted by our calculations can be found. However, at this stage, we are unable to reproduce this superdiffusivity quantitatively since Eq. (3) cannot be applied.

\section{Summary and conclusion}

We investigated particle propagation in pure two-dimensional and two-component turbulence theoretically. We demonstrated that the relation

$\Delta \mu(t)=-\frac{\Omega}{v B_{0}} \Delta \delta A_{z}(x(t), y(t))$

is a direct consequence of the equation of motion (NewtonLorentz-equation) in the case of magnetostatic and pure 2D turbulence. By applying Corrsin's independence hypothesis (Corrsin 1959, see Eq. (29)), we derived a general relation between the pitch-angle mean square deviation $\sigma_{\mu}^{2}(t)=\left\langle(\Delta \mu(t))^{2}\right\rangle$, and the characteristic function $\left\langle\cos \left(k_{x} \Delta x(t)+k_{y} \Delta y(t)\right\rangle\right.$. Obviously pitch-angle scattering can be described analytically if we have a realistic model available for perpendicular scattering.

By combining our general relation, Eq. (99), with QLT, we demonstrated that the pitch-angle diffusion coefficient $D_{\mu \mu}$ is equal to zero in the formal limit $t \rightarrow \infty$ (see Fig. 2). As soon as we considered a timescale $t$ at which the particle's orbit is closed, we obtained $\Delta \delta A_{z}(x(t), y(t))=0$, and it follows immediately that $\Delta \mu(t)=0$. These results confirm the previous investigations of Qin et al. (2006).

However, we were interested in the late-time behaviour of the parameters $\sigma_{\mu}^{2}(t)$ and $D_{\mu \mu}(t)$. In this limit, it was more convenient to replace the perpendicular motion of the charged particle by a stochastic motion. As a further assumption, we assumed a Gaussian distribution and employed the assumption that the perpendicular motion of the charged particles at late times can be replaced by the guiding-center motion. We demonstrated that we no longer find vanishing pitch-angle scattering. However, a subdiffusive behaviour of pitch-angle transport was obtained (see Eq. (60)). By setting $v=1$, which corresponds to a value of 2 of the inertial range spectral-index, we deduced a general formula for pitch-angle scattering (see Eq. (69)). This formula can be evaluated for different particle transport models such as a quasilinear model, stochastic Gaussian motion, non-Gaussian model.

The results for $D_{\mu \mu}^{2 \mathrm{D}}$ can easily be combined with the slab results obtained in previous articles to find new results for pitch-angle scattering in the well-known slab/2D composite model. As shown theoretically, after a certain timescale, the total pitch-angle Fokker-Planck coefficient approaches the slab limit. However, for low energetic particles, a long time has to be considered before the slab/2D result reaches diffusive behaviour. By comparing our theoretical result with simulations, we found a superdiffusive behaviour of parallel scattering. Since, this non-diffusivity was very weak, the weakly nonlinear theory of Shalchi et al. (2004b) was a good approximation valid for intermediate times, before the asymptotic QLT behavior was reached. It will be the subject of future work to replace Eq. (3) by a more general relation for describing parallel scattering also for non-diffusive pitch-angle scattering.

A fundamental problem of cosmic-ray scattering theory is the theoretical reproduction of observed parallel mean free paths (see e.g. Palmer 1982). A combination of QLT (see e.g. Bieber et al. 1994; Dröge 2003; Shalchi \& Schlickeiser 2004; Shalchi et al. 2006) and improved turbulence models, which includes the steepening of the turbulence spectrum at high wavenumber, dynamical turbulence effects, and spectral anisotropy, was used to reproduce parallel mean free paths of cosmic-rays inferred from solar energetic particle studies. On the other hand, QLT is, in general, invalid for parallel scattering: see Fig. 1 of this article. In this article, a possible explanation of this problem is provided: for medium timescales QLT is incorrect (see Fig. 3 of this article) since there is a strong scattering effect from $2 \mathrm{D}$ modes. Such timescales were considered in simulations, and, therefore, they cannot be reproduced by QLT and a nonlinear theory, such as the weakly nonlinear theory of Shalchi et al. (2004b), has to be applied. In the (formal) limit $t \rightarrow \infty$, however, the scattering contribution due to the $2 \mathrm{D}$ modes disappears and we find asymptotically the QLT result, which agrees with observations.

\section{Appendix A: Solving the integral and the series of QLT}

To describe pitch-angle scattering for the two-dimensional model within QLT, we investigate to following expression:

$$
\begin{aligned}
\sigma_{\mu}^{2}(t)= & 16 C(v) l_{2 \mathrm{D}} \delta B_{2 \mathrm{D}}^{2}\left(\frac{\Omega}{v B_{0}}\right)^{2} \\
& \times \int_{0}^{\infty} \frac{\mathrm{d} k_{\perp}}{k_{\perp}^{2}}\left(1+k_{\perp}^{2} l_{2 \mathrm{D}}^{2}\right)^{-v} \\
& \times \sum_{n=-\infty}^{\infty} J_{n}^{2}(W) \sin ^{2}(n \Omega t / 2) .
\end{aligned}
$$

By employing the integral-transformation $x=k_{\perp} l_{2 \mathrm{D}}$ and with

$a=\frac{R_{\mathrm{L}}}{l_{2 \mathrm{D}}} \sqrt{1-\mu^{2}}$ 
we find

$$
\begin{aligned}
\sigma_{\mu}^{2}(t)= & 32 C(v) \frac{l_{2 \mathrm{D}}^{2}}{R_{\mathrm{L}}^{2}} \frac{\delta B_{2 \mathrm{D}}^{2}}{B_{0}^{2}} \\
& \times \sum_{n=1}^{\infty} \sin ^{2}(n \Omega t / 2) \\
& \times \int_{0}^{\infty} \mathrm{d} x x^{-2}\left(1+x^{2}\right)^{-v} J_{n}^{2}(a x) .
\end{aligned}
$$

According to Gradshteyn \& Ryzhik (2000), the integral can easily be solved in the limit $a \rightarrow 0$

$$
\begin{aligned}
& \int_{0}^{\infty} \mathrm{d} x x^{-2}\left(1+x^{2}\right)^{-v} J_{n}^{2}(a x) \approx \frac{4^{-n}}{2} a^{2 n} \frac{\Gamma(n-1 / 2) \Gamma(v-n+1 / 2)}{[\Gamma(n+1)]^{2} \Gamma(v)} \\
& +\frac{1}{2} a^{2 v+1} \frac{\Gamma(n-v-1 / 2) \Gamma(v+1)}{\sqrt{\pi} \Gamma(v+3 / 2) \Gamma(3 / 2+n+v)} .
\end{aligned}
$$

Obviously the main contribution to the sum in Eq. (A.3) comes from $n=1$ and thus

$$
\int_{0}^{\infty} \mathrm{d} x x^{-2}\left(1+x^{2}\right)^{-v} J_{n}^{2}(a x) \approx \frac{a^{2}}{16 C(v)} \delta_{n 1}
$$

where we used $\Gamma(2)=1, \Gamma(1 / 2)=\sqrt{\pi}$, and Eq. (36). Thus, we find in the limit of small $a$

$\sigma_{\mu}^{2}(t)=2\left(1-\mu^{2}\right) \frac{\delta B_{2 \mathrm{D}}^{2}}{B_{0}^{2}} \sin ^{2}(\Omega t / 2)$.

The formal limit $a \rightarrow 0$ corresponds to $a=\sqrt{1-\mu^{2}} R_{\mathrm{L}} / l_{2 \mathrm{D}} \ll 1$ and thus to low energetic particles.

\section{Appendix B: Solving the integrals for the stochastic Gaussian model}

In this section of the Appendix, we solve the integral of Eq. (56) approximately. By using the parameter

$\gamma^{2}:=\frac{\sigma_{\perp}^{2}(t)}{2 l_{2 \mathrm{D}}^{2}}$

and the integral transformation $x=k_{\perp} l_{2 \mathrm{D}}$, we find

$\sigma_{\mu}^{2}(t)=\frac{8}{\pi} C(v) l_{2 \mathrm{D}}^{2} \delta B_{2 \mathrm{D}}^{2}\left(\frac{\Omega}{v B_{0}}\right)^{2} I\left(\gamma^{2}\right)$

with

$I\left(\gamma^{2}\right)=\int_{0}^{\infty} \frac{\mathrm{d} x}{x^{2}}\left(1+x^{2}\right)^{-v}\left[1-\mathrm{e}^{-\gamma^{2} x^{2}}\right]$.

According to Gradshteyn \& Ryzhik (2000), the integral can be expressed by confluent hypergeometric functions

$$
\begin{aligned}
I\left(\gamma^{2}\right)= & \frac{\sqrt{\pi} \Gamma(v+1 / 2)}{\Gamma(v)}\left[{ }_{1} F_{1}\left(-\frac{1}{2} ; \frac{1}{2}-v ; \gamma^{2}\right)-1\right] \\
& -\frac{1}{2} \Gamma(-v-1 / 2) \gamma^{2 v+1}{ }_{1} F_{1}\left(v ; v+\frac{3}{2} ; \gamma^{2}\right) .
\end{aligned}
$$

To proceed, we consider two limits of the parameter $\gamma^{2}$, namely $\gamma^{2} \ll 1$ and $\gamma^{2} \gg 1$.

\section{B.1. The limit $\gamma^{2} \ll 1$}

In this case, we can use (see e.g. Abramowitz \& Stegun 1974, page 504, Eq. (13.1.2))

$$
{ }_{1} F_{1}(a ; b ; z)=1+\frac{a}{b} z \ldots
$$

and thus Eq. (B.4) becomes

$I\left(\gamma^{2} \ll 1\right) \approx \frac{\sqrt{\pi} \Gamma(v-1 / 2)}{2 \Gamma(v)} \gamma^{2} \approx \frac{\gamma^{2}}{4 C(v)}$

where we used Eq. (36). Obviously Eq. (B.2) can be written as

$\sigma_{\mu}^{2}(t)=\frac{2}{\pi} l_{2 \mathrm{D}}^{2} \delta B_{2 \mathrm{D}}^{2}\left(\frac{\Omega}{v B_{0}}\right)^{2} \frac{\sigma_{\perp}^{2}(t)}{2 l_{2 \mathrm{D}}^{2}}$.

\section{B.2. The limit $\gamma^{2} \gg \infty$}

Here we can use (see Abramowitz \& Stegun 1974, page 508, Eq. (13.5.2))

$$
\begin{aligned}
{ }_{1} F_{1}(a ; b ; z)= & \frac{\Gamma(b)}{\Gamma(b-a)} \mathrm{e}^{ \pm \mathrm{i} \pi a} z^{-a}\left[1+o\left(z^{-1}\right)\right] \\
& +\frac{\Gamma(b)}{\Gamma(a)} \mathrm{e}^{z} z^{a-b} \sum_{n=0}^{\infty} \frac{(b-a)_{n}(1-a)_{n}}{n !} z^{-n} .
\end{aligned}
$$

By applying Eq. (B.8) onto Eq. (B.4) we find after lengthy straightforward algebra

$I\left(\gamma^{2} \gg 1\right) \approx \sqrt{\pi} \gamma$

and thus

$\sigma_{\mu}^{2}(t) \approx \frac{8}{\sqrt{\pi}} C(v) l_{2 \mathrm{D}}^{2} \delta B_{2 \mathrm{D}}^{2}\left(\frac{\Omega}{v B_{0}}\right)^{2} \gamma$.

\section{Appendix C: Integrals for $v=1$}

According to Eq. (70) pitch-angle scattering can be described by the formula

$$
\begin{aligned}
\sigma_{\mu}^{2}(t)= & 2\left(\frac{l_{2 \mathrm{D}}}{R_{\mathrm{L}}}\right)^{2}\left(\frac{\delta B_{2 \mathrm{D}}}{B_{0}}\right)^{2} \\
& \times\left(\frac{2}{\pi} \frac{r(t)}{l_{2 \mathrm{D}}}-1+I_{0}\left(\frac{r(t)}{l_{2 \mathrm{D}}}\right)-L_{0}\left(\frac{r(t)}{l_{2 \mathrm{D}}}\right)\right\rangle .
\end{aligned}
$$

In this section of the appendix, we consider extreme values for the ratio $r(t) / l_{2 \mathrm{D}}$.

\section{C.1. The limit $r(t) \ll I_{2 D}$}

In this case, we can employ (see Abramowitz \& Stegun 1974, Eqs. (12.2.1) and (9.6.10))

$L_{0}(z \ll 1) \approx \frac{2}{\pi} z+o\left(z^{3}\right)$

$I_{0}(z \ll 1) \approx 1+\frac{z^{2}}{4}$

to derive

$\left\langle\frac{2}{\pi} \frac{r(t)}{l_{2 \mathrm{D}}}-1+I_{0}\left(\frac{r(t)}{l_{2 \mathrm{D}}}\right)-L_{0}\left(\frac{r(t)}{l_{2 \mathrm{D}}}\right)\right\rangle \approx \frac{\left\langle r^{2}(t)\right\rangle}{4 l_{2 \mathrm{D}}^{2}}$

and therefore

$\sigma_{\mu}^{2}(t)=\frac{1}{2}\left(\frac{\delta B_{2 \mathrm{D}}^{2}}{B_{0}}\right)^{2} \frac{\left\langle r^{2}(t)\right\rangle}{R_{\mathrm{L}}^{2}}$. 


\section{C.2. The limit $r(t) \gg I_{2 \mathrm{D}}$}

In this case, we employ (see Abramowitz \& Stegun 1974, page 498, Eq. (12.2.6))

$L_{0}(z \gg 1)-I_{0}(z \gg 1) \approx-\frac{2}{\pi z}$

and thus

$\left\langle\frac{2}{\pi} \frac{r(t)}{l_{2 \mathrm{D}}}-1+I_{0}\left(\frac{r(t)}{l_{2 \mathrm{D}}}\right)-L_{0}\left(\frac{r(t)}{l_{2 \mathrm{D}}}\right)\right\rangle \approx \frac{2}{\pi} \frac{\langle r(t)\rangle}{l_{2 \mathrm{D}}}$

and therefore

$\sigma_{\mu}^{2}(t)=\frac{4}{\pi}\left(\frac{l_{2 \mathrm{D}}}{R_{\mathrm{L}}}\right)^{2}\left(\frac{\delta B_{2 \mathrm{D}}}{B_{0}}\right)^{2} \frac{\langle r(t)\rangle}{l_{2 \mathrm{D}}}$.

\section{Appendix D: The integrals for the modified wave spectrum in the energy range}

Equation (84) provides an analytic form for the parameter $\sigma_{\mu}^{2}$. In turn, we evaluate this form for $q<1$ and $x_{\min } \ll 1$. According to Gradshteyn \& Ryzhik (2000), this integral can be expressed by incomplete Gamma-functions $\Gamma(a, z)$

$$
\begin{aligned}
& \int_{x_{\min }}^{1} \mathrm{~d} x x^{-2-q}\left(1-\mathrm{e}^{-\gamma^{2} x^{2}}\right)+\int_{1}^{\infty} \mathrm{d} x x^{-2-2 v}\left(1-\mathrm{e}^{-\gamma^{2} x^{2}}\right)= \\
& \frac{x_{\min }^{-q-1}}{2(q+1)}\left[2-2 x_{\min }^{q+1}+(1+q) \Gamma\left(\frac{-q-1}{2}, \gamma^{2}\right)\left(\gamma x_{\min }\right)^{q+1}\right. \\
& \left.-(1+q) \Gamma\left(\frac{-q-1}{2}, \gamma^{2} x_{\min }^{2}\right)\left(\gamma x_{\min }\right)^{q+1}\right] \\
& +\frac{1}{2(2 v+1)}\left[2-(2 v+1) \gamma^{2 v+1} \Gamma\left(\frac{-2 v-1}{2}, \gamma^{2}\right)\right] .
\end{aligned}
$$

By taking into account the restriction

$1 \ll \gamma \ll x_{\min }^{-1}$

and by employing (see e.g. Abramowitz \& Stegun 1974)

$\Gamma(a, z \gg 1) \approx z^{a-1} \mathrm{e}^{-z} \rightarrow 0$

$\Gamma(a, z \ll 1) \approx \Gamma(a)-\frac{z^{a}}{a}+\frac{z^{a+1}}{a+1}$

this expression can be simplified to

$$
\begin{aligned}
\int_{x_{\min }}^{\infty} \mathrm{d} x \ldots= & x_{\min }^{-q-1}\left[-\frac{1}{2} \Gamma\left(\frac{-q-1}{2}\right)\left(\gamma x_{\min }\right)^{q+1}\right. \\
& \left.+\frac{1}{q-1}\left(\gamma x_{\min }\right)^{2}\right] .
\end{aligned}
$$

By employing $q<1$ and $x_{\min } \gamma \ll 1$, we find approximately

$$
\begin{aligned}
\int_{x_{\min }}^{\infty} \mathrm{d} x \ldots & =-\frac{1}{2} \Gamma\left(\frac{-q-1}{2}\right) \gamma^{q+1} \\
& =\frac{1}{q+1} \Gamma\left(\frac{1-q}{2}\right) \gamma^{q+1}
\end{aligned}
$$

where we used $\Gamma(z+1)=z \Gamma(z)$ from Abramowitz \& Stegun (1974), and thus for the parameter

$\sigma_{\mu}^{2}(t)=d_{1} \frac{l_{2 \mathrm{D}}^{2}}{R_{\mathrm{L}}^{2}} \frac{\delta B_{2 \mathrm{D}}^{2}}{B_{0}^{2}} \frac{1}{q+1} \Gamma\left(\frac{1-q}{2}\right) \gamma^{q+1}$.

Obviously we still find a subdiffusive behaviour as in the case of $q=0$.

Acknowledgements. This research was supported by the Deutsche Forschungsgemeinschaft (DFG) under the Emmy-Noether Programm (grant SH 93/3-1). As a member of the Junges Kolleg, A. Shalchi also acknowledges support by the Nordrhein-Westfälische Akademie der Wissenschaften. J. W. Bieber and W. H. Matthaeus acknowledge support of NASA grant NNX07AH73G.

\section{References}

Abramowitz, M., \& Stegun, I. A. 1974, Handbook of Mathematical Functions (New York: Dover Publications)

Bieber, J. W., Matthaeus, W. H., Smith, C. W., et al. 1994, ApJ, 420, 294

Carbone, V., \& Veltri, P. 1990, Geophys. Astrophys. Fluid Dyn., 52, 153

Cho, J., \& Lazarian, A. 2002, Phys. Rev. Lett., 88, 24, 245001

Cho, J., \& Lazarian, A. 2006, ApJ, 638, 811

Cho, J., Lazarian, A., \& Vishniac, E. T. 2002, ApJ, 564, 291

Corrsin, S. 1959, in Atmospheric Diffusion and Air Pollution, ed. F. Frenkiel, \& P. Sheppard (New York: Academic), 161

Dröge, W. 2003, ApJ, 589, 1027

Earl, J. A. 1974, ApJ 193, 231

Galtier, S., Nazarenko, S. V., Newell, A. C., \& Pouquet, A. 2002, ApJ, 564, L49

Goldreich, P., \& Sridhar, S. 1995, ApJ, 438, 763

Goldstein, M. L. 1976, ApJ, 204, 900

Gradshteyn, I. S., \& Ryzhik, I. M. 2000, Table of integrals, series, and products (New York: Academic Press)

Grappin, R. 1986, Phys. Fluids, 29, 2433

Horbury, T. S., Forman, M. A., \& Oughton, S. 2005, Plasma Phys. Control. Fusion, 47, B703

Jokipii, J. R. 1966, ApJ, 146, 480

Jones, F. C., Kaiser, T. B., \& Birmingham, T. J. 1973, Phys. Rev. Lett., 31, 485

Jones, F. C., Birmingham, T. J., \& Kaiser, T. B. 1978, Phys. Fluids, 21, 347

Le Roux, J. A., \& Webb, G. M. 2007, ApJ, 667, 930

Mace, J. W., Matthaeus, W. H., \& Bieber, J. W. 2000, ApJ, 538, 192

Matthaeus, W. H., Goldstein, M. L., \& Roberts, D. A. 1990, J. Geophys. Res., 95,20673

Matthaeus, W. H., Ghosh, S., Oughton, S., \& Roberts, D. A. 1996, J. Geophys. Res., 101, 7619

Matthaeus, W. H., Qin, G., Bieber, J. W., et al. 2003, ApJ, 590, L53

Matthaeus, W. H., Bieber, J. W., Ruffolo, D., Chuychai, P., \& Minnie, J. 2007, ApJ, 667, 956

Oughton, S., Priest, E. R., \& Matthaeus, W. H. 1994, J. Fluid Mech., 280, 95

Owens, A. J. 1974, ApJ, 191, 235

Palmer, I. D. 1982, Rev. Geophys. Space Phys., 20, 335

Qin, G., Matthaeus, W. H., \& Bieber, J. W. 2002a, Geophys. Res. Lett., 29

Qin, G., Matthaeus, W. H., \& Bieber, J. W. 2002b, ApJ, 578, L117

Qin, G., Matthaeus, W. H., \& Bieber, J. W. 2006, ApJ, 640, L103

Shalchi, A. 2005, Phys. Plasmas, 12, 052324

Shalchi, A. 2006, A\&A, 453, L43

Shalchi, A., \& Kourakis, I. 2007, A\&A, 470, 405

Shalchi, A., \& Schlickeiser, R. 2004, ApJ, 604, 861

Shalchi, A., Bieber, J. W., \& Matthaeus, W. H. 2004a, ApJ, 604, 675

Shalchi, A., Bieber, J. W., Matthaeus, W. H., \& Qin, G. 2004b, ApJ, 616, 617

Shalchi, A., Bieber, J. W., Matthaeus, W. H., \& Schlickeiser, R. 2006, ApJ, 642, 230

Shebalin, J. V., Matthaeus, W. H., \& Montgomery, D. 1983, J. Plasma Phys., 29, 525

Völk, H. J. 1973, Ap\&SS, 25, 471 